Rev Chil Salud Pública 2012; Vol 16 (2): $241-246$

Cifras de hoy en salud pública

\title{
La odontología que vivimos y no queremos: una crítica al escenario actual
}

\author{
THE DENTISTRY WE HAVE AND DON'T WANT: CRITICISM OF THE CURRENT SITUATION
}

\section{Escenario actual}

Actualmente en Chile, debido a la concepción dual de nuestro sistema salud, contamos con un sistema de salud público y otro privado, ambos regulados por el Ministerio de Salud. En odontología, el sector público de salud otorga las garantías explícitas de salud (GES) a sus beneficiarios (principalmente Fonasa $A$ y B) que, a la fecha, son la "Atención odontológica integral a niños de 6 años" desde el 2005, "Atención de urgencia dental ambulatoria " desde el año 2006, "Atención odontológica integral a adultos de 60 años" desde 2007 y "Atención odontológica integral de primigestas" desde el 2009. Junto a estas prestaciones se han consignado en las metas sanitarias para la década del 2000-2010 y 2010-2020 la priorización de atención a grupos específicos, como son los pacientes de 12 años y embarazadas no primigestas ${ }^{1-3}$. Debido a la alta demanda y a que la mayor parte de las jornadas laborales de los odontólogos se completan con los pacientes GES y grupos por metas negociadas año a año, la atención para el resto de la población es muy baja. Situación que minimiza la cobertura de la atención odontológica a un grupo muy reducido de la población.

En la atención privada, tenemos las consultas particulares y las dependientes de Isapres. Estas últimas han incluido en sus prestaciones convenios con clínicas privadas o servicios odontológicos dentro del mismo establecimiento, considerando -por supuesto- las prestaciones GES ya señaladas. Sin embargo, esto es aún insuficiente ya que la mayor parte de las prestaciones no se encuentran cubiertas y se debe hacer un copago; es decir, el tratamiento se reduce a lo que pueda pagar el propio bolsillo. Está de más señalar, que este sistema incluye todo tipo de tarjetas y formas de pago en crédito, que desgraciadamente excluyen a gran parte de la población de nuestro país que no cuenta con acceso a estos sistemas.

Por otra parte, tenemos el nuevo escenario del campo laboral en el que se encuentran los odontólogos. Este tema es complejo, debido en parte a la gran oferta universitaria para estudiar odontología, la inmigración de odontólogos y la escasa apertura de nuevos cupos laborales tanto en el sector público como privado. Esta situación ha dado paso a la aparición de las primeras generaciones de cirujanos dentistas desempleados, o empleados en forma parcial bajo el sistema de porcentaje en clínicas privadas dependientes de la demanda de servicios. Esto no ha sido abordado en investigaciones formales y sería interesante considerarlo, tal como se ha hecho para el análisis de oferta y demanda de médicos especialistas ${ }^{4}$. Sin embargo, es posible encontrar un sinnúmero de publicaciones o literatura gris al respecto (algunas serán citadas en el presente documento).

Según lo indicado a inicios del año 2012 por la Asociación Odontológica Argentina, utilizando como fuente al Colegio de Dentistas para los datos de Chile, existe un número total de odontólogos en nuestro país de 14.500. De estos, 2.590 corresponden a odontólogos extranjeros ${ }^{5}$. Actualmente, se ofrecen 
39 programas de Odontología en Chile, correspondiendo 9 a universidades públicas y 30 a instituciones privadas ${ }^{6}$. El total de odontólogos egresados en 2009 fue de 615, con un ascenso exponencial el año 2011, alcanzando un total de 1.200. En el mismo reportaje se indica que el porcentaje de la población de Chile que usa el servicio de atención pública corresponde a un $80 \%{ }^{5}$. Desgraciadamente, no se cita claramente la fuente de este porcentaje de cobertura, pues no es posible que corresponda al total real de acceso, pues como se ha señalado antes, los grupos priorizados no corresponden al $80 \%$ de la población total. En otras publicaciones en la red, asociado a páginas oficiales como la de ex dentistas generales de zona ${ }^{7}$, actual etapa de destinación y formación, las cifras son más dramáticas, pero poco claras (indican una proyección de un dentista por cada 600 habitantes para el año 2020). Según lo referido por estadísticas en reuniones de la Asociación de Clínicas Dentales, el año 2000 en Chile había un dentista cada 1.848 habitantes; en el año 2011 se estimó un dentista cada 1.133 habitantes y se proyecta que para el año 2016 con 12.000 estudiantes de Odontología (de primero a sexto año) habrá un dentista por cada 630 habitantes. Cousiño agrega en la misma columna que solo un $20 \%$ de la población tiene acceso a la odontología (fuente Instituto de Administración en Salud, dependiente de la Facultad de Ciencias Económicas y Administrativas U. de Chile); por cada dentista habrá un total de 126 pacientes potenciales. El año 2011 el Capítulo de Ejercicio Liberal, del Colegio de Dentistas de Chile, realizó un diagnóstico para cuantificar la situación de la odontología en Chile ${ }^{8}$. Los resultados estimados mostraron que existen 12.000 alumnos de $1^{\circ}$ a $6^{\circ}$ año, que estudian en 39 escuelas dentales de las distintas universidades existentes; de estas, solo 6 tienen acreditada la carrera. En otra columna del mismo autor se señala que existe una sobreorferta de un $43 \%$ de odontólogos a la fecha, con una proyección en los próximos 4 años, de una sobreoferta del $70 \%$ para el año 2016. Teniendo como antecedente que la OMS recomienda un dentista por cada 2.000 habitantes, para satisfacer las demandas mínimas de salud de la población ${ }^{8}$.

Esta sobreoferta ¿ha significado un mayor acceso de la población a atención dental? Cousiño estima que un 30\% tiene acceso; sin embargo, solo un $13 \%$ consulta al menos 1 vez al año a un dentista ${ }^{8}$. Según la Encuesta Nacional de Salud 2010- $2011^{9}$, el $27,8 \%$ de la población declara no haber visitado nunca al profesional odontólogo o no haberlo hecho dentro de los últimos 5 años desde la fecha de la última

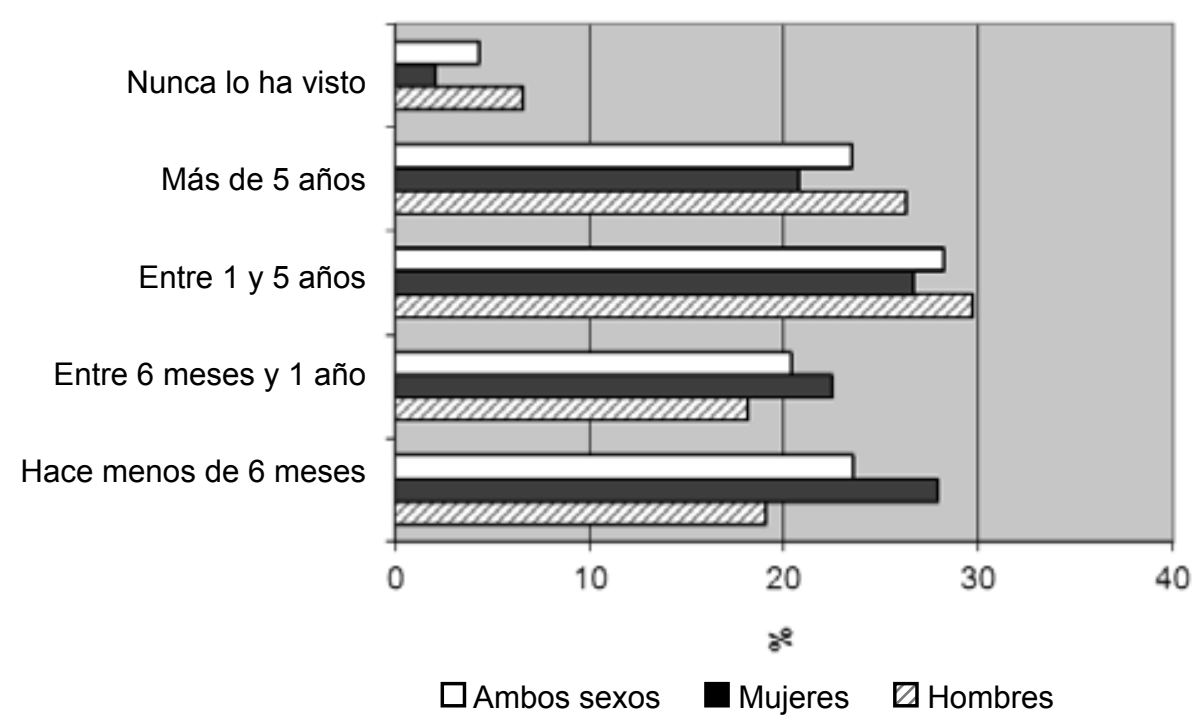

Figura 1. Distribución porcentual de las visitas al odontólogo según ENS Chile 2009 - 2010. 


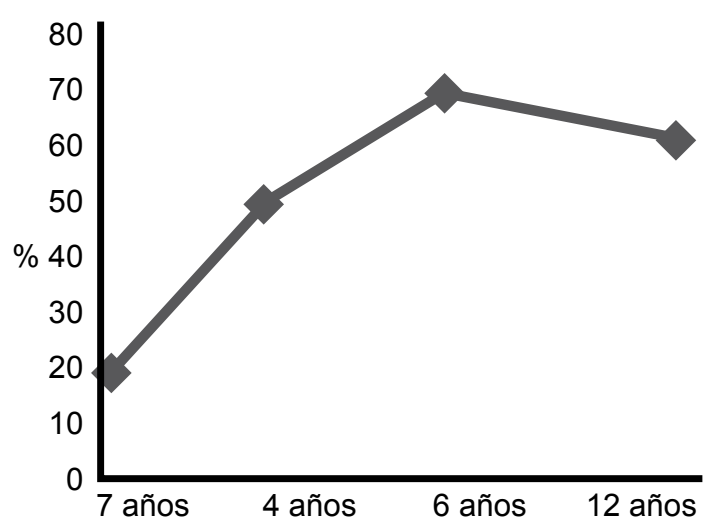

Figura 2. Prevalencia caries según diagnóstico de Salud

entrevista, con diferencias significativas según sexo $(32,9 \%$ en los hombres y $22,9 \%$ de las mujeres). El $4,3 \%$ de la población nunca ha tenido una evaluación por un profesional odontólogo, lo cual es similar en todos los grupos etarios. Por otra parte, el porcentaje de personas que no visita al odontólogo en los últimos 5 años, es mayor en los grupos de 45 a 64 años, y 65 y más años, en comparación con los participantes del grupo de 15 a 24 años. Mientras que en el estrato educacional alto el $15,1 \%$ de las personas declara no haber visitado nunca al odontólogo o no haberlo hecho en los últimos 5 años, en el nivel educacional medio este porcentaje aumenta al $27,5 \%$ y en el nivel bajo asciende, dramáticamente, a un 44,6\%. La muestra de personas cuya última visita al odontólogo ocurrió hace menos de 6 meses, es significativamente mayor en los participantes residentes en la zona urbana que en la zona rural $(24,7 \%$ y $16,2 \%$, respectivamente), mientras que el porcentaje que hace más de 5 años visitó al odontólogo es significativamente mayor en zona rural (Figura 1).

Pero este nuevo panorama ¿ha producido cambios epidemiológicos en la salud oral de los chilenos? ¿Ha mejorado la salud oral de la población chilena? Desgraciadamente, a pesar de que esto ha permitido un acceso más fácil a la atención dental, esperable por el aumento de la competencia, también ha significado una reducción de los costos de los tratamientos, en muchos casos, a expensas de la calidad del tratamiento. 0 la tendencia al sobretratamiento dental. Esta situación ya se ha estudiado en otros países, con una concepción de salud similar al nuestro, donde el sistema de salud se enfoca en la enfermedad y no en la salud ${ }^{10-14}$. Pero el factor más determinante es que el acceso se ve aún muy limitado a grupos específicos, ya que el costo en atención privada sigue siendo inalcanzable para la realidad nacional.

La prevalencia de caries en la población adulta en Chile según lo referido por Gamonal en 1996 , es de $99,2 \%$ para el grupo de 33 a 45 años y de un 99,4\% para el grupo de 65 a 74 años. En el estado de salud gingival se observa una alta prevalencia de individuos con pérdida de inserción clínica mayor a $3 \mathrm{~mm}$, en al menos uno de los dientes examinados. Las pérdidas de inserción mayores a $6 \mathrm{~mm}$, en al menos uno de los dientes examinados, fueron del $39 \%$ y $69 \%$ para los grupos de 35-44 años y 65-74 años, respectivamente $^{15}$. En el grupo de adultos de $35-44$ años solo un $20 \%$ conserva su dentadura completa mientras que este porcentaje baja a un $1 \%$ en los adultos de 65 a 74 años. La población de 35 a 44 años tiene un promedio de 6,5 dientes perdidos mientras en la población de 65 a 74 años este promedio es de 15,16-18.

Sin embargo, se podría pensar que la situación en población más joven es diferente. Al revisar los estudios de prevalencia de caries se puede observar los siguientes resultados. Como se observa en la Figura 2, la prevalencia de caries estimada a los 2 años es de un $16,8 \%$, a los 4 años de un $49,6 \%$, a los 6 años de un $70,4 \%$, a los 12 años de un $62,5 \%$ y a los 15 años de un $73,9 \%$. El problema de analizar solo la prevalencia es que dejamos de lado el sobretratamiento, esto es posible de estimar indirectamente el tratamiento por medio de la severidad de la historia de caries a través de los índices de ceod y COPD. Este ha tenido un descenso significativo en los últimos años en el grupo de 12 años, de 3,4 (1999) a 1,9 (2007). Sin embargo, al realizar el análisis por nivel socioeconómico es posible ver diferencias favorables para el grupo más acomodado económicamente ${ }^{16,17,19-21}$.

Pero, ¿es esto un problema de salud pública? Las condiciones orales representan el 1,4\% de la carga de enfermedad medida a través de años de vida ajustados por discapacidad (Avisa), correspondiendo a la patología o condición de salud número 18 para la muestra general. 
Las Figuras 3 y 4 muestran cómo este valor aumenta significativamente con la edad ${ }^{22}$. Según la ENS 2011, el 22,9\% de los encuestados usa próstesis dental (I.C. 95\%: 21,2 - 24,7). De los que usan prótesis dental, el 21,1\% se manifiesta "poco conforme" o "nada conforme, no me sirve" con la prótesis dental (I.C. 95\%: 17,8 - 24,7). El $25,3 \%$ de las personas declaró tener la percepción de necesitar el uso de prótesis dental (I.C. 95\%: $23,1-27,5)$, siendo este porcentaje muy similar para hombres y mujeres: $24,4 \%$ y $26,1 \%$, respectivamente.

\section{DISCUSIÓN}

Si por una parte tenemos la sobreoferta de odontólogos y, por otra, la alta morbilidad y carga de enfermedad que significan las patologías orales para la población chilena, ¿es posible dar solución al escenario actual? Esta situación es posible de solucionar con estrategias y políticas públicas que aseguren la oportunidad de acceso a atención dental en forma universal, por medio de una incorporación de este nuevo recurso humano a la atención pública de salud. Mientras siga existiendo una inequidad de acceso entre aquellos que pueden acceder a atención dental pagada y la gran mayoría de la población que no puede hacerlo, no lograremos disminuir la morbilidad de estas enfermedades y mucho menos sus secuelas.

Pero mejorar solo el acceso a atención no es suficiente, se debe poner un mayor énfasis en el enfoque preventivo, centrándonos en mantener a la población sana, libre de enfermedad, sien-

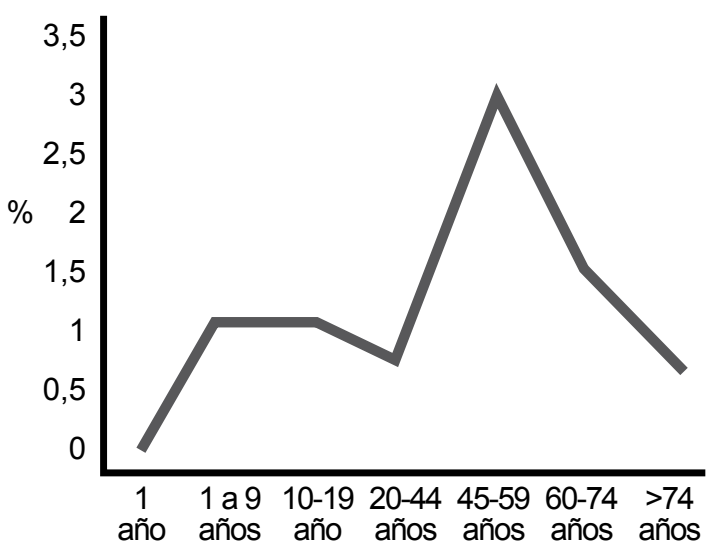

Figura 3. Porcentaje del total de Avisa.

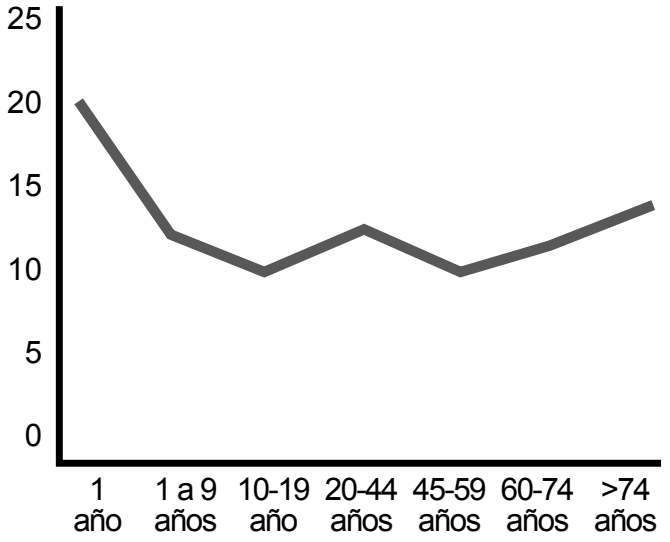

Figura 4. Posición de las condiciones orales del total de patologías

do verdaderos profesionales de la salud y no de la enfermedad. Solo así disminuiremos nuestra brecha de resultados en Salud oral, que es lo que realmente debe preocuparnos.

Sin embargo, este enfoque en el resultado nos obliga a analizar qué errores hay en el proceso que nos están impidiendo obtener lo esperado. Esto requiere un análisis más profundo dentro de las estrategias que se han implementado. Consideramos que uno de los determinantes más relevantes para mantener la salud oral en una población sana, o en una población tratada, es el control de salud. Pues es este que nos permite pesquisar a tiempo factores de riesgo y lesiones de caries incipientes, pudiendo llegar a tiempo para intervenir prematuramente a nuestros pacientes con medidas más simples, con un enfoque menos invasivo y con un menor costo. Es esta situación más costo- efectiva que rehabilitar a pacientes ya secuelados por caries, que no tuvieron acceso a un odontólogo previamente 0 aquellos que ya siendo tratados por el sistema no tuvieron continuidad de la atención. Esta continuidad de atención es un requisito obligado de enfermedades crónicas, como lo es la caries. Este punto es crucial y crítico; si sabemos abordarlo de forma eficiente y seria, creo que el panorama de la salud oral en Chile cambiaría absolutamente.

No podemos dejar de destacar los grandes hitos que se han hecho al respecto, en nuestro país. Partiendo por la incorporación del flúor al agua potable, estrategia de gran impacto si hablamos de medidas de prevención a alta escala, 
pero es aún insuficiente para la población con daño ya establecido. Por tanto, es necesario tomar medidas de dos frentes, uno que ya ha sido inicialmente abordado con el GES de 6 años y el componente odontológico del Control de niño sano, que es el enfoque preventivo en la población joven. $Y$ un segundo frente de limitación del daño ya establecido, por medio de una inyección de más odontólogos a los Servicios públicos de salud. Que nos permita abordar también, en forma eficiente el tema de la continuidad de tratamiento por medio de controles periódicos. Sabiendo que esto significa un alto costo de recurso humano e infraestructura, pero que los resultados responderían a una necesidad histórica en nuestra país y a las preferencias sociales de la población ${ }^{23}$.

Mi pregunta final es: ¿Cuál es el rol que debiesen cumplir las facultades de Odontología ante esta situación? Solo ellas... ¿O también debiesen intervenir otros actores?... Y: ¿Cuál es nuestra responsabilidad ante esta situación? Sin duda su participación como entes formadoras es crucial. Principalmente en el enfoque que hoy entregamos a nuestros alumnos. Es de suma importancia que nos centremos en la Salud como el actor principal y la prevención de la enfermedad, enfatizando que nuestra actividad no debe estar centrada en los procedimientos sino en una perspectiva más amplia que nos permita realmente mejorar la Salud de la población. Trabajando en conjunto con todas las entidades de salud, tanto públicas como privadas.

\section{Agradecimientos}

Agradezco las sugerencias al texto de las Dras. Patricia Moya y Araceli Raposo. Asimismo, la facilitación de los datos del Informe presente y futuro de la Odontología en Chile. 2011 (8) por parte del Dr. Sergio Cousiño.

\section{Referencias}

1. Ministerio de Salud. Gobierno de Chile [en línea]. [consultado en febrero de 2012]. Disponible en: http:// www.minsal.cl./portal/url/page/minsalcl/g_nuevo_ home/nuevo_home.html

2. Chile. Ministerio de Salud. Los objetivos sanitarios para la década 2000-2010. Evaluación de final del período: grado de cumplimiento de los objetivos de impacto. Santiago: Minsal; [s.f.].

3. Chile. Ministerio de Salud. Plan Nacional de Salud
2011-2020 para el cumplimiento de los Objetivos Sanitarios [en línea]. [consultado en enero de 2012]. Disponible en: http://bit.ly/SZLLJH

4. Banco Mundial, Chile. Ministerio de Salud. Estudio de brechas de demanda y oferta de médicos especialistas en Chile: informe final. 2009. Santiago: Minsal, Subsecretaría de Redes Asistenciales; 2010.

5. Asociación Odontológica Argentina. Jornadas Internacionales de la Asociación Odontológica Argentina [en línea]. [consultado en enero de 2012.]. Disponible en: http://www2.aoa.org.ar/Content.aspx?ld=9889

6. ADEO Chile: Número de Escuelas de Odontología en Chile [en línea]. [consultado en diciembre de de 2011]. Disponible en: http://blogadeo.blogspot.com/2011/02/ numero-de-escuelas-de-odontologia-en.html

7. Formas de ejercicio Profesional: «Veo Colegas Muertos» [el línea]. [consultado en noviembre de 2011]. Disponible en: http://formasdeejerciciodracerda. blogspot.com/2011/11/veo-colegas-muertos.html

8. Colegio de Cirujano Dentistas de Chile. Capítulo Ejercicio liberal. Informe presente y futuro de la Odontología en Chile. Santiago: Colegio de Cirujanos Dentistas de Chile; 2011.

9. Chile. Ministerio de Salud. Departamento de Epidemiología; Pontificia Universidad Católica. Departamento de Salud Pública; Universidad Alberto Hurtado. Observatorio Social. Encuesta Nacional de Salud: Chile 2009-2010. Santiago: Minsal.

10. Baelum V. What is an appropriate caries diagnosis? Acta Odontol Scand. 2010;68(2):65-79.

11. Simonsen RJ. Overtreatment? You bet it is! J Esthet Restor Dent. 2007;19(5):235-6; discussion 237.

12. Jin EY. Overtreatment. Dent Today. 2000;19(8):6.

13. Hasegawa TK Jr, Matthews M Jr. Overtreatment or appropriate treatment? Tex Dent J. 1995; 112(5):37$9,41$.

14. Elderton RJ. Overtreatment with restorative dentistry: when to intervene? Int Dent J. 1993:43(1):17-24.

15. Gamonal J. Prevalencia de enfermedades periodontales y de caries dental en la población de 35-44 y de 65 a 74 años de nivel socioeconómico bajo y mediobajo de la provincia de Santiago, Región Metropolitana, y determinación de los recursos humanos necesarios para su tratamiento. Rev Fac Odontol Univ Chile. 1996;14(1):56-57.

16. Soto L, Tapia R, Jara G, Rodríguez G. Diagnóstico Nacional de Salud Bucal del Niño de 6 Años. Santiago: Minsal; 2007.

17. Soto L, Tapia R, Jara G, Rodríguez G, Urbina T, Venegas $C$, et al. Diagnóstico Nacional de Salud Bucal del Adolescente de 12 años y Evaluación del Grado de Cumplimiento de los Objetivos Sanitarios de Salud Bucal 2000- 2010. Chile: Universidad Mayor; 2007.

18. Gamonal J, Mendoza C, Espinoza I, Muñoz A, Urzúa I, Aranda W, et al. Clinical attachment loss in Chilean adult population: First Chilean National Dental Exami- 
nation Survey. J Periodontol. 2010 Oct;81(10):140310.

19. Urbina T, Caro JC, Vicent M. Caries dentaria y fluorosis en niños de 6 a 8 y 12 años de la II, VI, VIII, IX, X y RM. Chile 1996. Santiago: Minsal. División Programas de Salud. Departamento Odontológico; 1996.

20. Ceballos M, Acevedo C, Corsini G, Jans A. Diagnóstico Nacional de Salud Bucal de niños de 2 y 4 años que asisten a la educación preescolar en la. Región Metropolitana. Chile: Minsal; 2007.
21. Hoffmeister L, Moya P, Benadof D y col. Implementación Clínica del problema de salud con Garantías Explícitas en Salud: Salud Oral Integral para niños(as) de 6 años. Chile; 2008.

22. Chile. Ministerio de Salud. Estudio de carga de enfermedad y carga atribuible. Chile: Minsal; 2007.

23. Chile. Ministerio de Salud. División Planificación Sanitaria. Estudio de preferencias sociales para la definición de Garantías Explícitas en Salud. Chile: Minsal; 2008. 